\title{
Nonholistic processing in mental rotation: Some suggestive evidence
}

\author{
JOHN C. YUILLE and JAMES H. STEIGER \\ University of British Columbia, Vancouver, British Columbia V6T IWS, Canada
}

\begin{abstract}
A series of experiments, using a modification of the Shepard and Metzler mental rotation task, was performed to investigate Shepard's "holistic rotation" hypothesis. Effective figural complexity was manipulated in the experiments in two distinct ways. In one manipulation, blocks were added to the standard 10-block figures. In the other manipulation, the figures used and the direction of angular rotation were restricted so that some featural information in the figures was redundant, that is, unnecessary for the discrimination task at hand. There were two major conclusions. First, when figural complexity is effectively manipulated, it has a powerful effect on the "speed of mental rotation," as measured by the slope of the curve relating reaction time to angular disparity. Second, it is possible, by ignoring featural redundancy, to construct experimental paradigms in which "complexity" of figures is apparently manipulated but has no effect on speed of mental rotation. This fact provides a possible explanation of why some previous experiments have failed to find a complexity effect in mental rotation.
\end{abstract}

During the past decade, the historic debate about nature of mental representation of visual information has been joined with renewed vigor (e.g., Anderson, 1978; Kosslyn \& Pomerantz, 1977; Paivio, 1975; Pylyshyn, 1973, 1979; Yuille \& Catchpole, 1977). One currently controversial issue is whether representational processes are analog, that is, whether the form of the internal representation bears an isomorphic correspondence to the object or event represented.

There is considerable support for the analog position (e.g., Glass, Holyoak, \& Santa, 1979). Some of the most frequently cited evidence comes from the extensive research by Shepard and his colleagues on "mental rotation." Using pairs of block figures like those in Figure 1, Shepard and Metzler asked their subjects to compare the two figures in a pair and to determine, as quickly and accurately as possible, whether they were the same (differing only in angular orientation) or different (mirror images of each other). The critical finding in the research was that the latency required to make a "same" judgment is a linear function of the angular disparity between the two figures. Shepard and his co-workers have argued that this finding, together with related results, indicates that the comparison between the two figures is carried out by mentally rotating one of the figures into a position corresponding to that of the other figure. This mental rotation is assumed to be an analog of the

This research was supported by grants to both authors from the National Science and Engineering Research Council of Canada (A327 and A4640) and from the University of British Columbia. The order of authors is arbitrary, and reprint requests can be obtained from either author, c/o Department of Psychology, University of British Columbia, 2075 Wesbrook Mall, Vancouver, B.C. V6T IW5, Canada. actual rotation of an object of this form, and the mental act of rotation is assumed to take real time. In keeping with this view, Shepard has used the slope of the regression line relating reaction time to angular disparity to estimate the "speed of mental rotation."

By 1976, a number of variations of the original
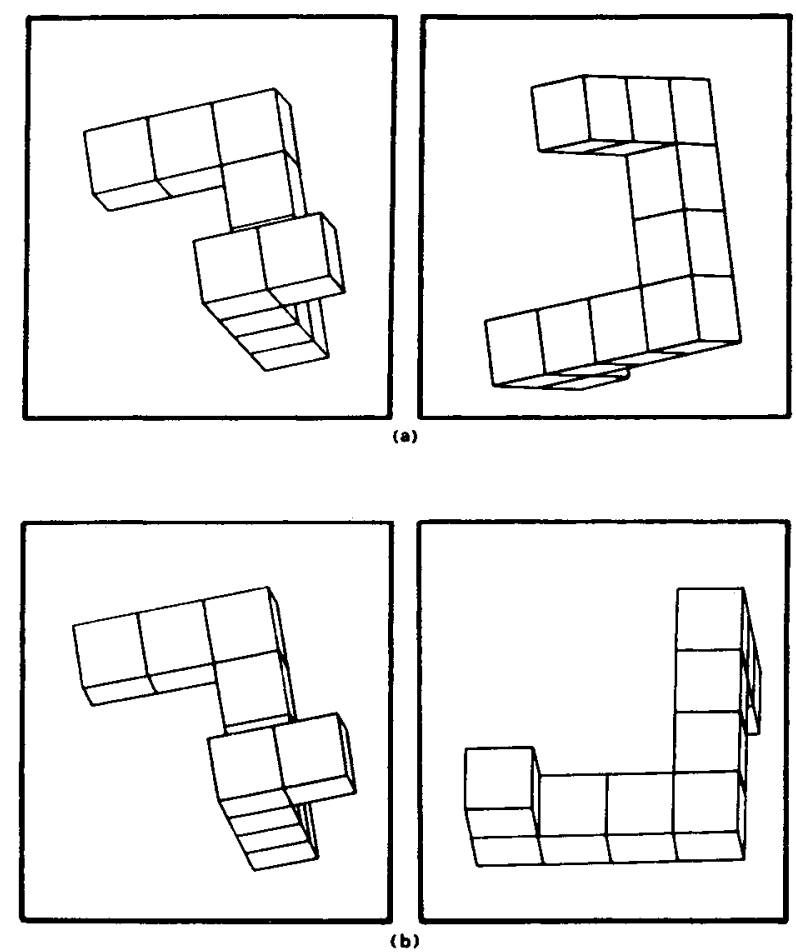

Figure 1. Experiment 1: Standard three-dimensional block figures (from Shepard \& Metzler, 1971). 
"simultaneous presentation" paradigm had been investigated. Cooper and Shepard (1978), summarizing this work, found an impressive unanimity in the papers they surveyed. Almost without exception, the data seemed to support the "holistic, analog" view of mental representation. However, others, notably Pylyshyn (1973), have suggested that these theoretical inferences are not appropriate. Though none would contest the fact that "mental rotation" paradigms yielded monotonically increasing, and often linear, reaction time curves, there is some doubt about what such curves imply about the underlying mental representation. Indeed, Anderson (1978) has expressed doubts about the possibility of any unambiguous resolution of the ongoing debate.

Stating that the "dependence of rotation time on object complexity is predicted by all models which assume that the internal representation of a visual object is operated upon serially, piece-by-piece, or feature-by-feature," Cooper and Shepard (1978) stressed the absence of a complexity effect in mental rotation as important evidence in favor of the "holistic, analog" view. However, they cited only one study (Cooper \& Podgorny, 1976) that had examined complexity effects and failed to find them. This study had used figures (random polygons) that were quite different from those of Shepard and Metzler (1971). Interestingly, a seemingly obvious extension of the original Shepard-Metzler experiment to similar, but more complex, block drawings had not yet been attempted. Pylyshyn (1979), using figures and experimental paradigms that differed appreciably from those of Cooper and Podgorny, found fairly strong complexity effects.

Pylyshyn's (1979) paper raised doubts about the status of complexity effects in mental rotation, and also voiced strong disagreement with the "holistic, analog" view. However, the numerous differences in stimulus materials and experimental arrangements between Pylyshyn's work and that of Shepard and his.co-workers has left some ambiguity about how these discordant data should be interpreted. While there is no obvious reason why mental rotation should be object-dependent, the fact remains that any modification or qualification of Shepard's hypothesis would be on firmer ground when based on a research analog of the typical Shepard paradigm.

Using block drawings like those used by Shepard and Metzler in a "memory" mental rotation task, Steiger and Yuille (Note 1) produced evidence that raised further doubts about the "holistic" hypothesis. In the memory task, subjects were required to memorize a "standard" block figure in a particular orientation. Then they were shown a series of figures, one figure at a time, and they had to judge whether each was the same as the memorized figure (differing only in orientation) or a mirror image. If the subjects were shown both the standard figure and its mirror image during the memorization, they were able to respond more rapidly to the comparison figures than when only the standard was presented during the memorization phase. Clearly, in this mental rotation task, information about the relationship between figures to be discriminated had an impact on performance.

In a within-subject comparison of performance in the "memory" and "simultaneous" paradigms, Steiger and Yuille found much faster estimated rotation speeds (and much lower error rates) in the "memory" condition. Specifically, estimated "rotation speed" was $487 \mathrm{deg} / \mathrm{sec}$ in the "memory" condition and a fairly typical $95 \mathrm{deg} / \mathrm{sec}$ in the "simultaneous" condition. In attempting to explain this rather large discrepancy in performance, Steiger and Yuille concluded that a "piecemeal processing" hypothesis was most in line with their data (e.g., some subjects reported using a segment-comparison strategy).

The present series of experiments seeks to extend the work of Pylyshyn (1979) and Steiger and Yuille (Note 1) to provide a more direct examination of the holistic processing hypothesis. Ironically, a previously unnoticed aspect of the Shepard-Metzler block figures provides a basis for just such an examination. Specifically, when rotation is restricted about the vertical axis, featural information in only the bottom half of the figure is sufficient to determine whether they are the same or different. The forms in Figure 2, which are the bottom halves of the Shepard and Metzler
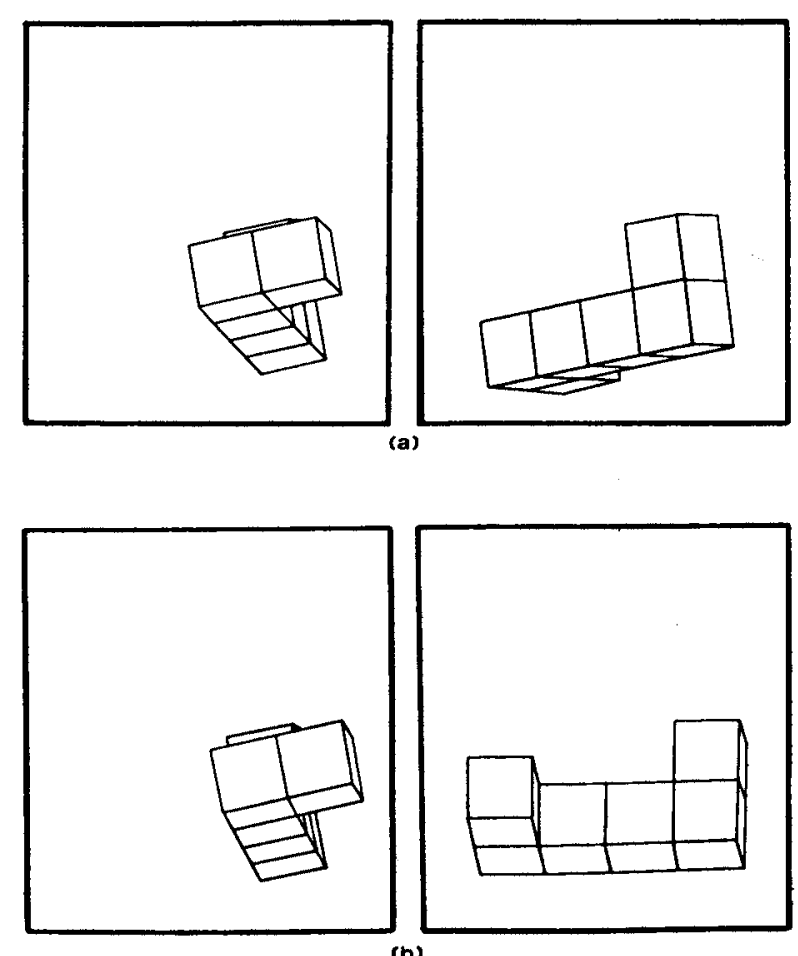

(b)

Figure 2. Experiment 1: Bottom halves of standard figures. When rotation is restricted about vertical axis, featural information in the bottom half is sufficient to determine whether figures are the same or different. 
figures (Figure 1), amplify this point. When the horizontal arm at the bottom of one figure is rotated into congruence with the same arm of the other figure, the single protruding cube determines figural congruence. Hence, in processing the Shepard-Metzler figures, there is really no need to look at the tops of the figures at all.

The redundancy of the top part of each figure provides an especially convenient means of exploring the role of complexity in mental rotation, because it allows a manipulation of effective figural complexity without changing the figures displayed to the subjects. In the first experiment, we compared the mental rotation performance of subjects who were informed about the redundancy (i.e., told that they need examine only the bottom half of each figure) with the performance of subjects who remained uninformed.

\section{EXPERIMENT 1}

\begin{abstract}
Method
Subjects. The subjects were 33 undergraduate volunteers, each of whom received $\$ 5$ for their participation. Nine of these subjects exceeded a preestablished criterion of $10 \%$ errors and were dropped from the experiment, so the final sample consisted of 24 individuals (12 females).

Materials. The materials were adapted from those used by Shepard in his previous work and by Steiger and Yuille (Note 1). The stimulus materials were block figures of the kind represented in Figure 1. Twelve rotated versions of each figure were generated, consisting of figures rotated around the vertical axis in orientations of 0 through $330 \mathrm{deg}$, in $30-\mathrm{deg}$ increments. In addition, the 12 mirror-image versions of each of these figures were drawn. As noted above, the top half of each of those figures is redundant in solving the comparison task. All figures were produced on the computer graphic facility at the University of British Columbia, and were photographed on color film and mounted as $35-\mathrm{mm}$ slides. Duplications of the basic 24 figures allowed multiple instances of each figure. Pairs of these slides were randomly generated to produce combinations differing in orientation by 0 through $180 \mathrm{deg}$ in 30-deg steps. These pairings included figures that were the same and pairs that were mirror images of one another. Thus, all possible combinations of same and different figure pairs in seven angular disparities were generated.
\end{abstract}

Apparatus. The pairs of block figures were projected using two Model AF-2 Kodak Carousel slide projectors. The figures were rear-projected on a screen $.96 \mathrm{~m}$ from the projectors and $1 \mathrm{~m}$ in front of the subject. The presentation of a pair of figures, initiated by a switch operated by the experimenter, triggered a photoelectric cell that started a timer. There were two switches in front of the subject (one labeled "same," the other labeled "different"), and pressing either of them stopped the timer and removed the slides.

Procedure. The subjects were tested individually. Each subject was assigned randomly to one of two groups. Subjects in both groups were instructed about the general nature of the task and were shown sample figures. Then they each received 168 trials, each trial involving the presentation of a pair of figures. The task for the subject was to press one switch if the two figures were the same (differing only in terms of an angular rotation) or a second switch if the two figures were different (being mirror images). These trials represented 12 instances of each combination of seven angular disparities for both same and different pairs of figures. Upon completion of these trials, the subject was given a questionnaire inquiring about the strategies he/she used in performing the task. At this point, each subject assigned to the experimental group was instructed concerning the part-figure solution to the task.
They were informed that an inspection of the bottom half of the figure was sufficient to solve the task. This was followed by an additional 168 trials of paired figures conducted in the same fashion as the first set of trials. A second questionnaire was completed when the second set of trials finished. This second questionnaire duplicated the first, and also asked whether the subjects' strategy had changed after the first trial set. One subject whose questionnaire indicated that he had failed to understand the experimental instructions was dropped from the study. Control subjects rested after the first questionnaire, performed the second 168 trials, and then completed the second copy of the questionnaire.

\section{Results}

The analyses in this report are atypical in two respects. First, we chose the median of each subject's reaction times at each rotation angle as the unit of analysis, since the median is less sensitive than the mean to the inherently high variability stemming from the use of naive undergraduates in relatively brief experimental sessions. The typical group curves relating reaction time to rotation angle were then constructed using the means of these medians, for each rotation angle. Second, we computed regression lines and "estimated rotation speeds" for each subject in order to supplement the (more typical) analysis of group data. The individually based rotation speeds revealed rather high variability. For example, estimated rotation speeds prior to training ranged from 31.8 to $272.3 \mathrm{deg} / \mathrm{sec}$ for the experimental group.

Overall error rates were higher for experimental subjects than for controls in both test sets, although differences were not statistically significant. Error rates for experimental subjects were $6.10 \%$ before the midperiod and $3.22 \%$ after. For controls, the rates were $4.31 \%$ and $1.93 \%$.

The basic question in the analysis is, "Did the redundancy instructions significantly affect rotation speed in the experimental group, relative to the control group?" The answer is an unqualified "yes," and is corroborated by both individual and group data. The essential outcome is demonstrated in the regression lines for the reaction time data for both experimental and control groups. These regression lines, for curves of reaction time (to "same" figures) vs. angular disparity, are given in Figures 3 and 4.

The key aspect of these figures is the fact that, while slope changes only slightly for the controls (Figure 3 ), there is a major change for the experimental group. "Rotation speeds," estimated from the inverse of the slope of the reaction-time curves, are $71.6 \mathrm{deg} /$ $\mathrm{sec}$ for the control-before and $60.4 \mathrm{deg} / \mathrm{sec}$ for the experimental-before groups. After the midperiod, the control group increased slightly to $87.8 \mathrm{deg} / \mathrm{sec}$, while the experimental group improved radically, to $143.1 \mathrm{deg} / \mathrm{sec}$.

Analysis of group means for the subjects' individually calculated "rotation speeds" corroborates the findings from the group data. Before the midperiod, the mean slopes were 85.90 and $84.45 \mathrm{deg} / \mathrm{sec}$ for the experimental and control groups, respectively. 
After the break, the values were $180.66 \mathrm{deg} / \mathrm{sec}$ for the experimental group and $112.13 \mathrm{deg} / \mathrm{sec}$ for the control. The difference in mean change between ex-

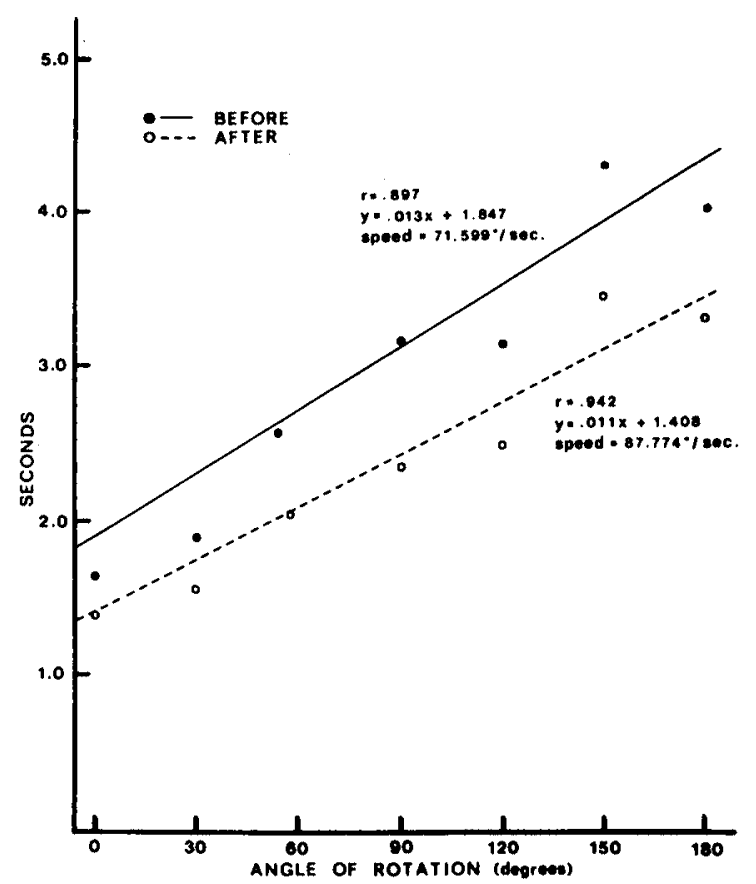

Figure 3. Experiment 1: Reaction time as a function of angle of rotation for the control group. No information regarding featural redundancy was given between trial sessions.

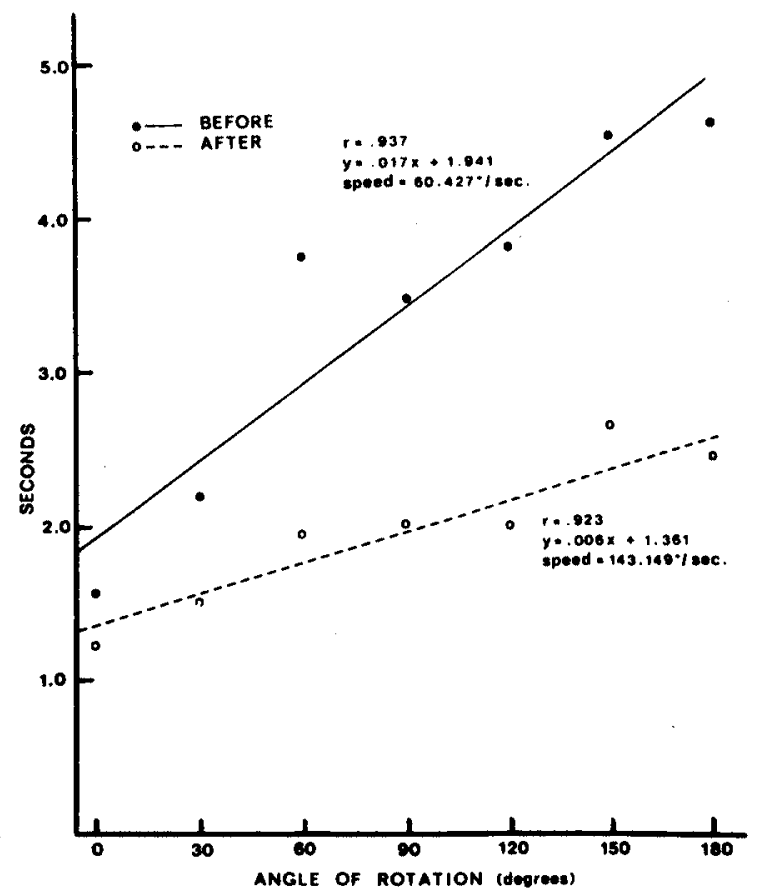

Figure 4. Experiment 1: Reaction time as a function of angle of rotation for the experimental group. Subjects were informed of featural redundancy after completing the first trial session. perimental $(94.76 \mathrm{deg} / \mathrm{sec})$ and control $(27.68 \mathrm{deg} /$ $\mathrm{sec}$ ) groups is statistically significant $[\mathrm{t}(22)=3.11, \mathrm{p}=$ $.01]$ and large enough to provide rather strong evidence for the differential effect of experimental instructions.

\section{Discussion}

This experiment used the standard Shepard-Metzler " $A$ " figures. Key information for discriminating the standard figures from their mirror images is concentrated in the bottom half of the figure, in the sense that the figures may be reliably differentiated by examining the bottom halves alone, while they may not be discriminated by examining only the top halves. Hence, the subject who performs an exhaustive comparison of the features of the two figures is, from a sequential feature-analytic perspective, wasting both time and effort. Accordingly, any change in strategy that eliminates the processing of the noninformative features should improve performance. On the other hand, a holistic rotation hypothesis, without (in the present context) capacity limitations, would predict little, if any, improvement in performance from such a strategy change.

The results clearly indicate that although estimated "rotation speeds" increased significantly for both experimental and control groups, the improvement was significantly greater for the experimentals, whose "rotation speeds" doubled.

It should be noted that Shepard and his colleagues have generally used a mixture of figure types in their research, rather than a homogeneous set like that employed in the present research. Their procedure probably precluded both the experimenters and subjects in previous research from discovering the featural redundancy that we exploited here. However, notice should also be taken of the fact that, perhaps surprisingly, subjects in our untrained control group, who received only one kind of figure over a sequence of trials, apparently did not discover the redundancy in these figures spontaneously. One possible explanation for this finding is that subjects search only for a simpler strategy beyond some level of figural complexity at which an inherent capacity limitation makes exhaustive, piecemeal comparison impossible. The complexity of Shepard-Metzler figures may not reach that level.

In the experiments reported below, we examined further the relationships among figural complexity, subject strategy, and performance in the "mental rotation" task. These experiments are unusual in that, unlike previous studies on the effect of complexity on rotation performance, they used figures similar, and in several cases identical, to those used by Shepard and Metzler (1971) in their original experiment. More complex figures were created by adding more blocks to the standard figures. We found that, indeed, complexity, when properly manipulated, has a very strong 
effect on mental rotation performance. We also discovered why some types of "complexity manipulation" are unlikely to affect mental rotation performance parameters, thus suggesting a resolution of the apparent discrepancy between the results of Cooper and Podgorny (1976) and those of Pylyshyn (1979).

\section{EXPERIMENT 2}

\section{Method}

Subjects. Eighteen student volunteers were each paid $\$ 5$ for their participation in the experiment. Each subject was required to make less than $10 \%$ errors in classifying pairs of figures. Eight of the subjects exceeded this criterion, leaving 10 subjects (five females) in the final sample.

Materials. The materials consisted of block figures like those demonstrated in Figure 5. Notice that the bottom 10 blocks of these figures constitute the standard Shepard-Metzler figures (Figure 1). Thus, an additional seven blocks have been added to the top of the figure employed in Experiment 1. The figure comparison task can still be solved by examining only the bottom arm (five blocks) of each figure. A standard figure, designated SO, and its mirror image, designated MO, were generated, as were rotated versions of each of these for angular disparities between 30 and $330 \mathrm{deg}$ from the originals, in steps of $30 \mathrm{deg}$. The 24 figures were drawn on the computer graphics facility of the University of British Columbia, traced on bond, photographed, and mounted as $35-\mathrm{mm}$ slides. Duplication of these slides permitted multiple instances of each of the figures. Eighty-four random pairings of these figures were made, with the restriction that half of the pairs contained the same figure type, differing only in angular rotation. The angular difference was between 0 and $180 \mathrm{deg}$, in 30-deg steps, with six replications of each angular disparity. For the remaining pairs, the two figures were mirror images differing in the same fashion in terms of angular displacement. Thus, there were six instances of each combination of figure pair type and angular rotation. The apparatus was identical to that used in Experiment 1.

Procedure. All subjects in this experiment received standard instructions essentially the same as the control subjects in the first experiment; that is, they were not informed of the figural redundancy. After 28 practice trials, each subject received 168 trials, involving two presentations of the 84 pairs of slides. Following the rotation task, the subjects completed a questionnaire concerning the strategy they had employed.

\section{Results}

The high percentage $(44 \%)$ of subjects who had to be rejected for making more than $10 \%$ errors is substantially more than we have encountered in previous research on mental rotation. Clearly, the complex figures present a more challenging and difficult task to subjects than the simpler Shepard-Metzler figures that we have used in the past.

For those subjects who were able to pass the $10 \%$ criterion, median response times at each rotation angle were found for their correct responses on trials in which figures were the same. The means across subjects of these results are presented graphically in Figure 6. For the sake of comparison, the figure includes a typical finding, taken from Steiger and Yuille (Note 1), obtained on the same apparatus with the simpler Shepard-Metzler figures.

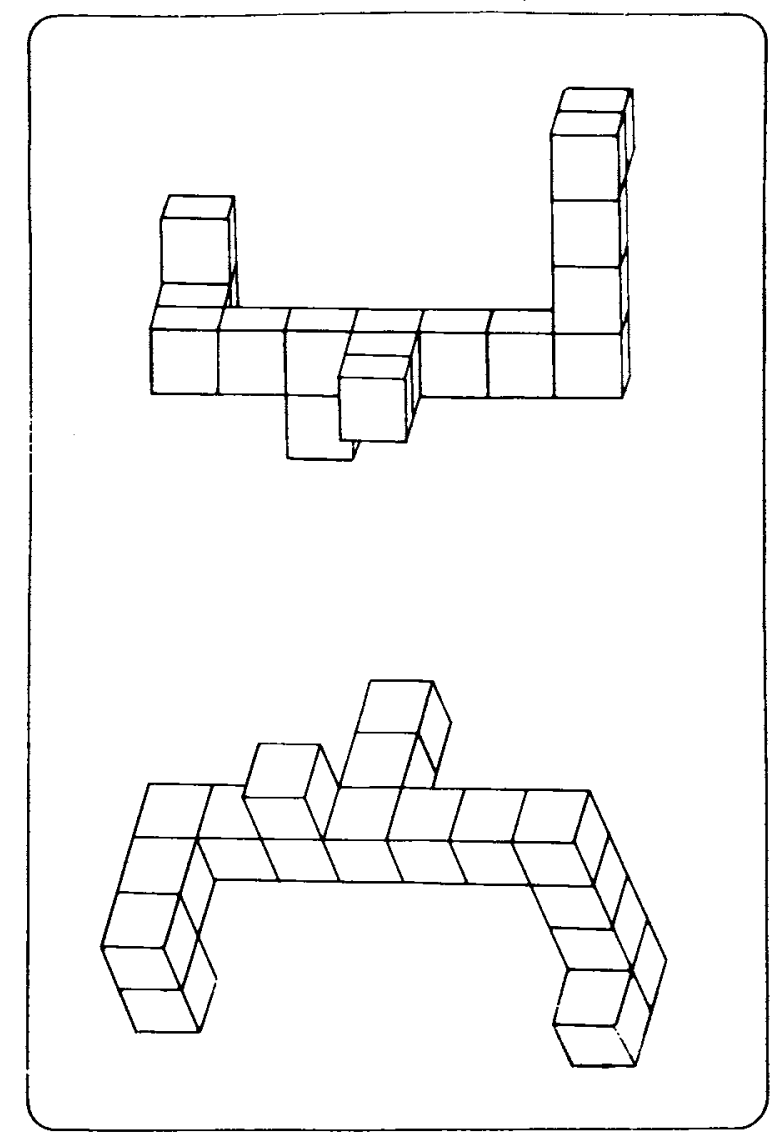

Figure 5. Experiment 2: Complex block figures. Bottom 10 blocks constitute the standard Shepard-Metzler figures. Comparison task is solvable by examining only the bottom arm of each figure.

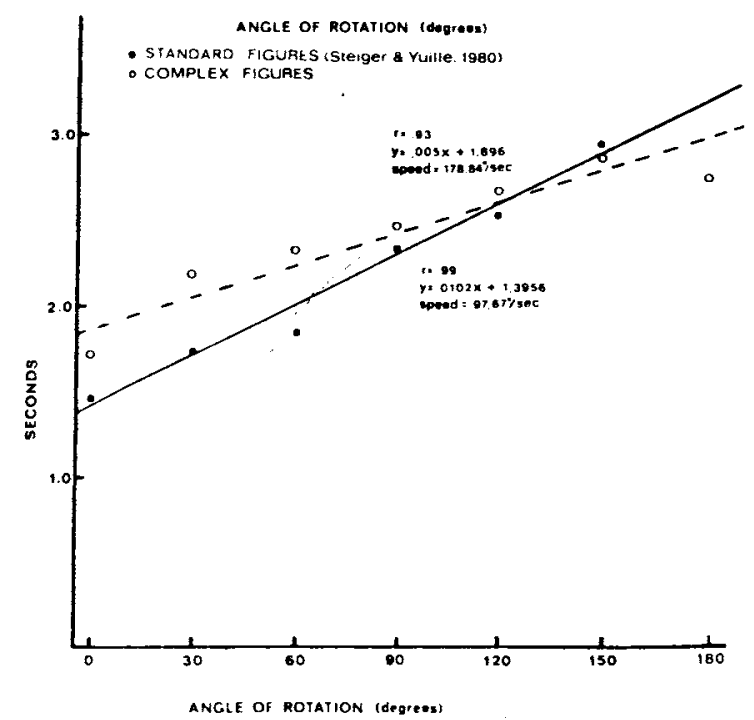

Figure 6. Experiment 2: Reaction time as a function of angle of rotation. Comparison task speeds for subjects vlewing standard and complex figures are shown. 


\section{Discussion}

It is apparent from Figure 6 that subjects passing criterion were able to "mentally rotate" the complex figures about as fast as typical subjects would rotate the simpler figures. In fact, the angular disparity had less effect with complex figures than with simple ones. This result seems surprising, but the postexperimental questionnaire produced an obvious explanation. The complexity of this task places considerable stress on the subject's processing capabilities. As noted earlier, over $40 \%$ of the subjects found the demand sufficiently great that they committed more than $10 \%$ errors. Our hypothesis is that this increased demand led to a search for an alternative strategy, one simpler and more manageable than that employed with the simple figures. This search led, in turn, to the discovery, and exploitation, of featural redundancy. Hence, after a brief period of adjustment, "successful" subjects began to solve the problem by paying attention to only a segment of each figure. This led to the apparently anomalous result that "rotation speed" was actually faster $(179 \mathrm{deg} / \mathrm{sec})$ than typical performance with simpler figures. The spontaneous discovery of redundancy did not occur in Experiment 1 because the figures used in that experiment were not complex enough to mandate a search for a simplification strategy.

There is an alternative explanation for the relatively fast reaction times to complex figures in Experiment 2. This result could demonstrate that there is no effect of complexity on mental rotation, when ShepardMetzler-type figures are employed. Thus, the results of the second experiment could provide support for the Cooper and Shepard (1978) conclusion. The contrast between these two explanations (no complexity effect vs. spontaneous discovery of redundancy) requires examination. The purpose of the third experiment was to carry out such a contrast by removing the redundancy inherent in the larger figures.

To this end, we employed a revised and enlarged figure set. The new figure set included the same large figures used in the previous experiment, but also contained figures with some segments twisted 90 deg out of alignment with the other segments. An example of a "twisted" figure is given in Figure 7.

Notice that the two figures again contain the standard figures (the bottom 10 blocks). Also, 7 blocks have been added, as in Experiment 2. However, in this case five blocks in the middle of the left figure have been rotated $90 \mathrm{deg}$. Our figure set also included cases in which the top ( 4 top blocks), middle (as in Figure 7), or bottom (bottom 5 blocks) of the figure was twisted by $90 \mathrm{deg}$. The inclusion of these twisted figures, which "match" on some features but not on others, means that subjects attempting to compare each pair of figures on the basis of partial featural information (e.g., bottom 5 blocks) will commit errors

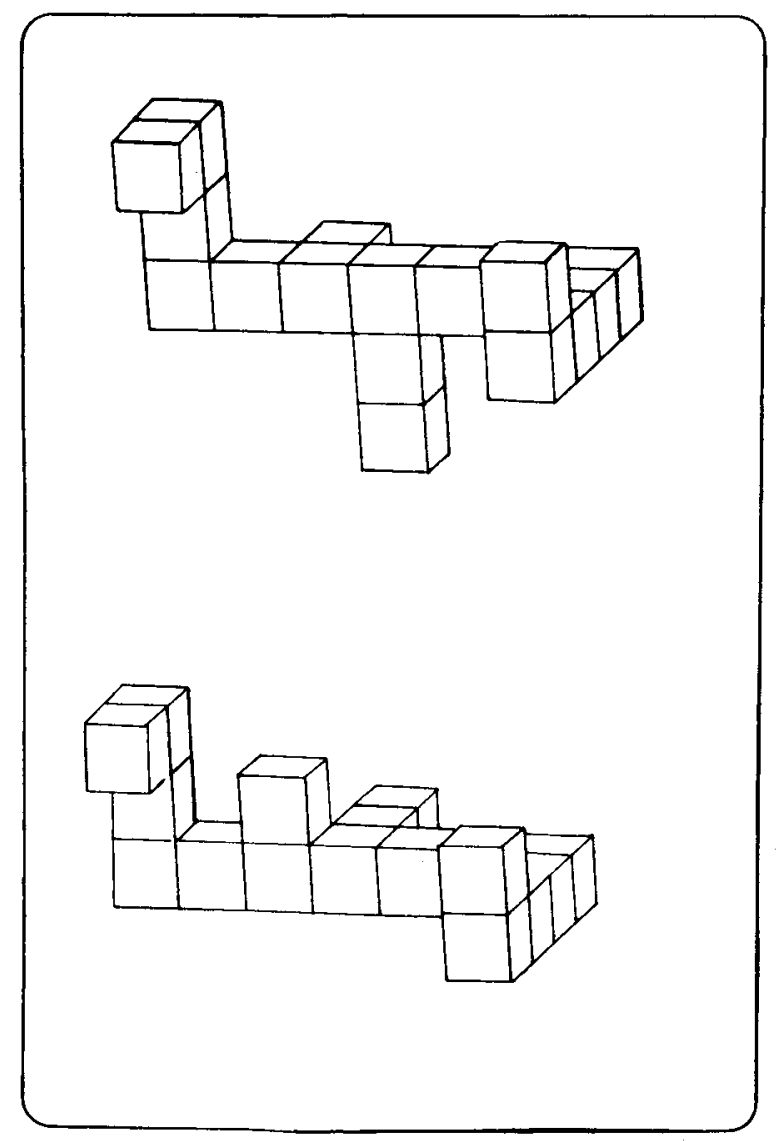

Figure 7. Experiment 3: Sample "twisted" figure. Middle segment is rotated 90 deg out of alignment with other segments.

on some of the twisted figures. Alternatively, the presence of the twisted segments in some figures might preclude the search for redundancy, and if subjects do make piecemeal comparisons, they will require longer to respond than subjects in the previous experiment.

\section{EXPERIMENT 3}

\section{Method}

Subjects. Thirteen volunteers were each paid $\$ 5$ for their participation. In this experiment, which proved difficult for most subjects, we were forced to modify our rejection criterion. The subjects were permitted to err on up to $25 \%$ of their responses, and 10 (five males) of the 13 subjects tested met this criterion.

Materials. The materials consisted of the same slides as those employed in Experiment 2, plus a set of additional slides generated by twisting the top, middle, or bottom sections of the figures by 90 deg. A total of 168 pairs of figures were generated so that half of them were the same, differing only in angular rotation. It should be noted that in some cases each of a pair of figures contained the same twist in the center or other segment of the figure. Half of the figure pairs were different. Half of the "different" pairs were different by virtue of being mirror images of one another, while half were different because of a segment twist. Different pairs contained a wide variety of angular rotations.

Procedure. The procedure was exactly as in Experiment 2. 


\section{Results}

Means of subjects' median response times for correct identification of "same" pairs of figures are plotted in Figure 8. For the sake of comparison, these are plotted together with the data from Experiment 2.

These data indicate that the procedural modification, using the twisted figures, was extremely effective. Subjects required a considerable amount of time to decide whether the two figures were the same, in fact longer than in any research that we have done using mental rotation paradigms. The estimated speed of mental rotation is $33 \mathrm{deg} / \mathrm{sec}$, less than one-quarter of that reported in Experiment 2.

As noted above, we also found that the subjects made more errors (an average of $11.49 \%$ ) than we had found in our previous research, requiring us to change our criterion for retention of subjects' data. Analysis of the errors among those subjects that we retained showed that the vast majority occurred when slides were different because of a segment twist. For example, error rate on "different-twisted" trials was $19 \%$, while error rate on "different-mirror" trials was only $3 \%$. If the "mental rotation" task simply involved the internal manipulation of a visual image of one of the figures to determine its congruence or incongruence with the other figure, it should make no difference whether the figures are different by virtue of being mirror images or by virtue of a segmental difference between them. The combined data from Experiments 2 and 3 demonstrate clearly that

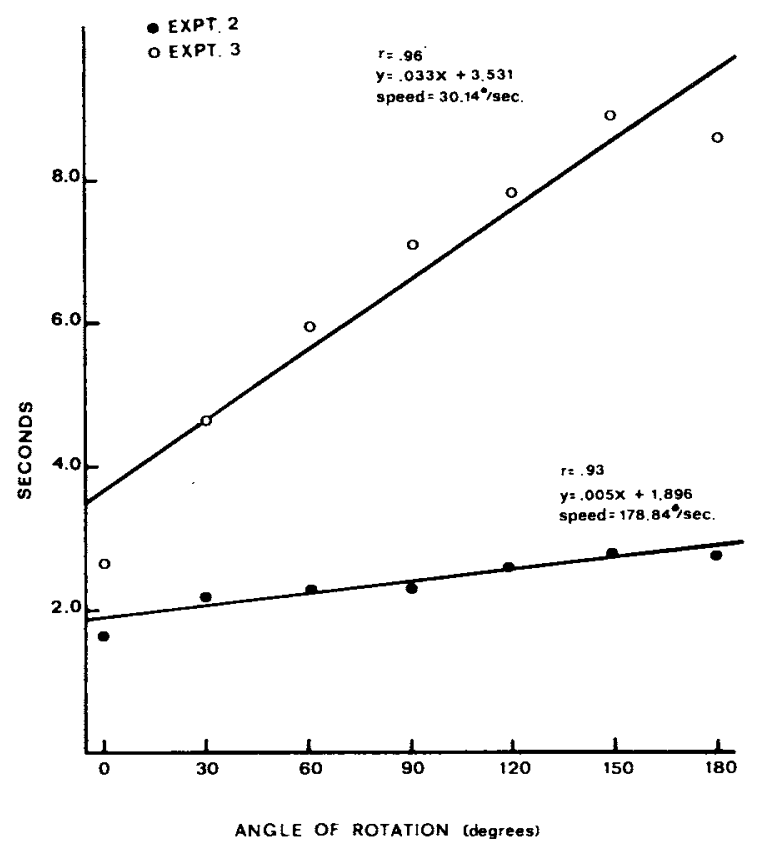

Figure 8. Experiment 3: Reaction time as a function of angle of rotation. Rotation speed using figures with figural redundancy (Experiment 2) is compared with rotation speed achieved by subjects viewing figures with redundancy eliminated. the type of "different" figures employed ("mirror" or "twisted") makes a considerable difference, not only on the performance on "different" trials, but also on "same" trials.

\section{EXPERIMENT 4}

Given the importance of the preceding findings to hypotheses concerning mental imagery, it seemed appropriate to carry out a replication. Comparison of Experiments 2 and 3 was between subjects, which, perhaps, compromises the interpretation of the results slightly. In this experiment, we combine the experimental manipulations of Experiments 2 and 3 in a within-subject design counterbalanced for order.

\section{Method}

Twenty-five volunteers were each paid $\$ 10$ for their participation in the experiment. Sixteen subjects (eight females) met our criteria of less than $10 \%$ errors in the "mirror-only" condition (analogous to Experiment 2) and less than $25 \%$ errors in the "twisted and mirror" condition (analogous to Experiment 3).

Materials. The materials consisted of the same slides, in new random pairings, that were employed in Experiments 2 and 3.

Procedure. Each subject engaged in the experiment on 2 consecutive days. On one day the subject was asked to compare complex figures of the type used in Experiment 2. On the other day, the procedure was the same as in Experiment 3. Half of the subjects served in the "mirror-only" condition (analogous to Experiment 2) on the first day, while half served in the "twisted and mirror" (analogous to Experiment 3) on the first day.

\section{Results}

Averaged median latencies for the two conditions are plotted in Figure 9.

Note that these data have been collapsed over the order in which the conditions were received. The order of conditions proved to be irrelevant. Subjects who received the "mirror-only" condition on the first day had an average estimated "speed of mental rotation" of $105.7 \mathrm{deg} / \mathrm{sec}$ on these figures, while subjects who received the "mirror-only" condition on the second day had an average rotation speed of $112.06 \mathrm{deg} / \mathrm{sec}$. Similarly, for the twisted segment condition, subjects receiving this first averaged $64.50 \mathrm{deg} / \mathrm{sec}$ estimated rotation speeds, while those receiving this condition second averaged $58.96 \mathrm{deg} / \mathrm{sec}$.

Thus, the pattern of results was essentially the same regardless of presentation order. Overall, subjects "rotate faster" in conditions when twisted figures are not present. This duplicates the most important finding in Experiments 2 and 3. On the other hand, estimated rotation speeds were notably faster in these experiments than in the analogous conditions in Experiments 2 and 3 . This is not solely attributable to the extended experience of the subjects in the withinsubject design of this experiment, and probably relates to variability in our subject populations.

The error data confirm that the subjects found the twisted condition to be more difficult than the non- 


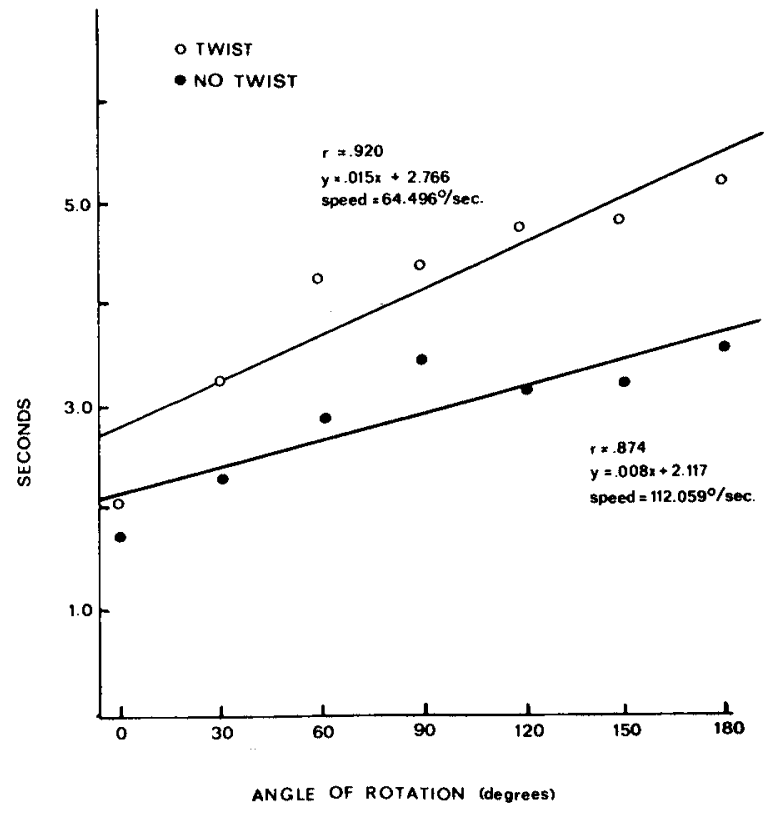

Figure 9. Experiment 4: Reaction time as a function of angle of rotation. Subjects viewed mirror/no-twist task one day, mirrortwist another day. "Rotation" speed was faster when twisted figures were not present.

twisted one. On "different" trials, subjects made substantially more errors $(9.41 \%)$ in the "twistedmirror" condition than in the "mirror-only" condition $(4.17 \%)$. It should also be noted that, overall, 14 of the 16 subjects committed more errors in the "twisted-mirror" than in the "mirror-only" condition.

Although the behavioral differences between groups of subjects as a function of order of presentation of two conditions were minimal, it is interesting to note that there were differences in subjective reports of strategies employed in the sessions. Of the eight subjects who received the "mirror-only" condition first, six reported that they noticed a change from the first to the second session. In other words, they were aware of the addition of the twisted figures. In contrast, only one of the eight subjects who received the "twisted-mirror" condition first noted a change when shifted to the "mirror-only" condition.

\section{GENERAL DISCUSSION}

The four experiments reported here have provided converging evidence that appears to resolve the role of complexity in the so-called mental rotation task. The results of the first experiment demonstrated that a reduction in the complexity of the standard ShepardMetzler figures (by the use of instructions concerning featural redundancy) allows more rapid figural comparisons. When the subject is informed that he/she need examine only part of each figure, overall performance, including estimated "rotation speed," is reliably faster. This finding supports the hypothesis that subjects may mediate this task by a piecemeal comparison of figure segments, rather than by holistic rotation. This hypothesis received additional support when the standard Shepard-Metzler figures were made more complex. The third and fourth experiments provided a demonstration that increasing the complexity of the Shepard-Metzler figures decreases the speed of the figural comparison task. There is clearly a complexity effect in this task.

In the first experiment, complexity was varied through the use of instructions concerning the inherent redundancy of the standard figures. It is interesting that the subjects who did not receive these instructions did not spontaneously discover the redundancy, while in Experiment 2, in which the more complex block figures were used, those subjects who could perform the task did discover the feature redundancy in the figures. Apparently, whether subjects spontaneously seek simplification of the block figures depends on the number of features in the figures. There is a level of complexity beyond which subjects are forced to simplify, but below which they will use all of the features (even if redundant) to solve the task.

The notion of a processing limit in the performance of this task is consistent with a feature analysis or piecemeal interpretation of mental rotation. Our hypothesis is that the mental rotation task is performed by a series of sequential comparisons of figure segments. The subject selects one segment of a figure (presumably one arm) and compares it with the corresponding segment in the other figure. If correspondence can be found between the two segments, the subject must retain some information concerning the orientation change required to achieve congruence. The information concerning the change in segment orientation must be temporarily stored, and a second segment of the two figures compared. This process continues until the entire figure has been examined. The limitation in performing the task would be the number of segments to be searched. If too many segments are involved, perhaps too much information must be temporarily retained (exceeding the shortterm memory capacity of the subject). Alternatively, manipulating too many segments might simply be too confusing. In either case, when the number of segments to be compared becomes too large, the subject must either simplify the task by reducing the number of segments attended or perform poorly. Both outcomes were evident in the present experiments. It should be noted that the eye-movement data reported by Carpenter and Just (1978) are consistent with this "piecemeal" interpretation of mental rotation. They observed that subjects compared segments of the block figures in a sequential manner.

The current results suggest that the failure of Cooper and Podgorny (1976) to find a complexity effect stems 
from their inadequate manipulation of figural complexity. Their stimulus figures were based upon the random nonsense forms generated by Attneave and Arnoult (1956). Complexity is defined in this set of figures by the number of points determining inflections on the perimeter of each figure. While this may be an appropriate definition of the perceptual complexity of the forms in some context, it is inappropriate when pairs of forms are being compared. The more complex forms contain distinguishing features so that the entire figure does not have to be examined when comparing it with a second figure. The distinguishing feature(s) is sufficient, and additional features are redundant. Thus, the "more complex" figures do not necessarily differ in effective complexity in the comparison task. This may well explain why Cooper and Podgorny (1976) did not find a complexity effect in their experiment.

\section{REFERENCE NOTE}

1. Steiger, J. H., \& Yuille, J. C. Long term memory and mental rotation. Manuscript submitted for publication, 1980.

\section{REFERENCES}

Anderson, J. R. Arguments concerning representations for mental imagery. Psychological Review, 1978, 85, 249-277.
Attneave, F., \& Arnoult, M. D. The quantitative study of shape and pattern perception. Psychological Bulletin, 1956, 53, 452-471.

Carpenter, P. A., \& Just, M. A. Eye fixations during mental rotation. In J. Senders, R. Monty, \& D. Fisher (Eds.), Eye movements and psychological process II. Hillsdale, N.J: Erlbaum, 1978.

Cooper, L. A., \& Podgorny, P. Mental transformations and visual complexity processes: Effects of complexity and similarity. Journal of Experimental Psychology: Human Perception and Performance, 1976, 2, 503-514.

Cooper, L. A., \& Shepard, R. N. Transformations on representations of objects in space. In E. C. Carterette \& M. Friedman (Eds.), Handbook of perception (Vol. 8). New York: Academic Press, 1978.

Glass, A. L., Holyoak, K. J., \& Santa, J. L. Cognition. Reading, Mass: Addison-Wesley, 1979.

Kosslyn, S. M., \& Pomerantz, J. R. Imagery, propositions, and the form of internal representations. Cognitive Psychology, 1977, 9, 52-76.

Paivio, A. Perceptual comparisons through the mind's eye. Memory \& Cognition, 1975, 3, 635-647.

Pylyshyn, Z. W. What the mind's eye tells the mind's brain: A critique of mental imagery. Psychological Bulletin, 1973, 80, 1-24.

PyLYSHYN, Z. W. The rate of "mental rotation" of images: A test of a holistic analogue hypothesis. Memory \& Cognition, 1979, 7, 19-28.

Shepard, R. N., \& Metzler, J. Mental rotation of threedimensional objects. Science, 1971, 171, 701-703.

Yuille, J. C., \& CATch pole, M. J. The role of imagery in models of cognition. Journal of Mental Imagery, 1977, 1, 171-180.

(Manuscript received February 2, 1981;

revision accepted for publication September 22, 1981.) 